\title{
Viscoelasticity Measurement of Heart Wall in in vivo
}

\author{
Hiroshi Kanai \\ Department of Electronic Engineering, Graduate School of Engineering, Tohoku University, \\ Sendai 980-8579, Japan. Email: hkanai@ecei.tohoku.ac.jp
}

\begin{abstract}
By measuring spatial distribution of the minute vibrations in the heart wall from the chest wall using ultrasound, we find that some impulses propagate along the heart wall in healthy human subjects just after closure of the aortic valve for the first time. Their amplitude is found to be on the order of several tens of micrometers, and up to $100 \mathrm{~Hz}$. Their propagation speed shows frequency dispersion, which agrees with the theoretical characteristics of the Lamb wave. The instantaneous viscoelasticity of the wall is then noninvasively determined. These findings have a novel potential for myocardial tissue characterization in clinical diagnosis.
\end{abstract}

\section{INTRODUCTION}

Magnetic resonant imaging [1], [2], computer tomography [3], and conventional ultrasonography enable clinical visualization of cross-sectional images of the human heart, but their imaging is restricted to large motion $(>1 \mathrm{~mm})$ and low frequency components $(<30 \mathrm{~Hz})$. Analysis of the motion-mode (M-mode) image [4], [5] — the magnitude of the sequentially obtained radio frequency (RF) data acquired in conventional echocardiography-is a candidate for transthoracic measurement of the heart-wall motion. However, the detectable amplitude is still greater than the wavelength, which is equal to $410 \mu \mathrm{m}$ for ultrasound with a frequency of 3.75 MHz. The tissue Doppler imaging technique [6][10] — modified ultrasound two-dimensional (2-D) color flow mapping - enables us to acquire motion distribution of the myocardium. Even in this measurement, however, the sampling frequency of the displayed motion of the heart wall is low (at most $60 \mathrm{~Hz}$ ). All these previous clinical methodologies, therefore, are employed to measure only large $(>0.4 \mathrm{~mm})$ and slow $(<30 \mathrm{~Hz})$ motion due to the heartbeat which can be recognized by medical doctors with the naked eye.

By artificially actuating shear waves in tissues or phantoms, their propagation speed and/or viscoelasticity can be determined for tissue characterization [11]-[17]. Yet spontaneously actuated vibrations propagating in the heart wall, which differ from electrically excited waves [18], [19], have not been recognized at all.

In this paper, using a newly developed method to measure spatial distribution of the minute vibrations in the heart wall from the chest wall using ultrasound [20], we find that some impulses propagate along the heart wall in healthy human subjects just after closure of the aortic valve for the first time and their propagation speed shows frequency dispersion, which agrees with the theoretical characteristics of the Lamb wave [21], [22].
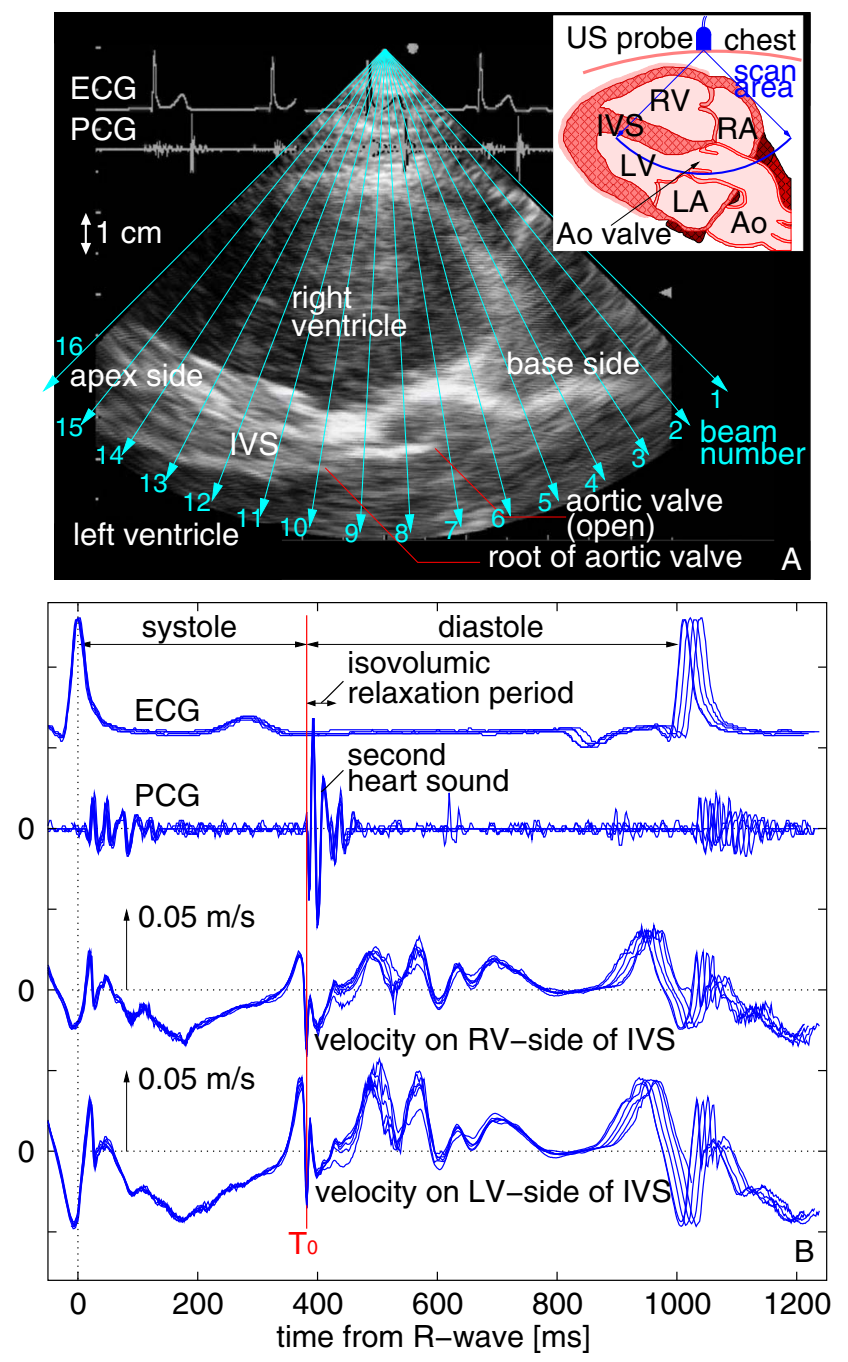

Fig. 1. (A) A cross-sectional image measured by a conventional ultrasound diagnosis system for a young healthy male. The upper-right illustration shows the scanning range of the ultrasonic beams in this imaging. (B) In vivo measurement results for the healthy man in Fig. A at two points set along the 13th ultrasonic beam. Each waveform for six consecutive cardiac cycles was overlaid. The timing of the aortic-valve closure is denoted by $T_{0}$. (LV: left ventricle, LA: left atrium, RV: right ventricle, RA: right atrium, US probe: ultrasonic probe, IVS: interventricular septum, Ao: aorta, ECG: electrocardiogram, PCG: phonocardiogram (heart sound).) 


\section{In Vivo EXPERIMENTS}

Figure 1A shows a typical cross-sectional image of a heart obtained by conventional echocardiography for a young healthy subject from the transthoracic parasternal longitudinalaxis view. The upper-right illustration shows the scanning range of the ultrasonic beams in this imaging. From the motion picture of Fig. 1A, the large slow motion during one heartbeat can indeed be recognized.

\section{A. Distribution of the Vibrations in and on the Heart Wall}

To measure the original vibrations of the heart sounds audible by stethoscope, we have developed an ultrasoundbased transthoracic method to directly measure the heart wall vibrations [23]-[25]. For this measurement, the sampling frequency (the time resolution) should be increased from the $30 \mathrm{~Hz}$ in conventional echocardiography to about $500 \mathrm{~Hz}$. To realize this, the number of the transmitted directions of the ultrasonic beams was decreased from 240 to 16 as shown in blue arrows of Fig. 1A. For all of the multiple points preset at $75-\mu \mathrm{m}$ intervals in the heart wall along 16 ultrasonic beams, the vibrations were simultaneously measured as a waveform with a sampling frequency of $450 \mathrm{~Hz}$ for the first time by the newly developed phased tracking method [23], [24]. Figure 1B shows a typical example of the vibrations on both sides - the right ventricular (RV) side and left ventricular (LV) side-of the interventricular septum (IVS) for the subject in Fig. 1A. Though 6 consecutive heartbeats were overlaid, small, rapid vibrations were measured with high reproducibility, although they cannot be observed by any conventional equipment. As shown by these waves, some discriminative impulses can be observed, especially at end-systole. At end-systole, a slow upward pulse continued for about $30 \mathrm{~ms}$ before the aorticvalve $(\mathrm{AV})$ closure timing $T_{0}$. A steep downward impulse then occurred at $T_{0}$ for the beginning of the isovolumic relaxation (IR) period [26].

By applying the same method to about 160 points preset at the $\mathrm{AV}$ closure timing $T_{0}$ at equal intervals of $770 \mu \mathrm{m}$ in the IVS along each of the 16 scan lines, the vibration was simultaneously obtained for each point during the short-period between $T_{0}-35 \mathrm{~ms}$ to $T_{0}+35 \mathrm{~ms}$. The occurrence of dips was gradually delayed from the root of AV to the apex-side by several milliseconds. To the contrary, a steep upward impulse propagated from the root of $\mathrm{AV}$ to the base-side of the heart.

\section{B. Propagation Speed of the Impulse Along the Heart Wall}

Since the wavelength of the detected impulsive wave is about $10 \mathrm{~cm}$ for a $30 \mathrm{~Hz}$ component and is comparable to that of the heart wall, its propagation phenomenon cannot be visualized by showing the amplitude distribution of the resultant impulsive wave along the IVS. Instead, the phase value varies from 0 to 360 degrees in one wavelength. In this paper, therefore, 2-D spatial distribution of the instantaneous phase values of the measured wave for a selected frequency component from $10 \mathrm{~Hz}$ to $100 \mathrm{~Hz}$ is color-coded [20]. Figure 2 shows this distribution for a $60 \mathrm{~Hz}$ frequency component. For this imaging, the short-time Fourier transform was applied to

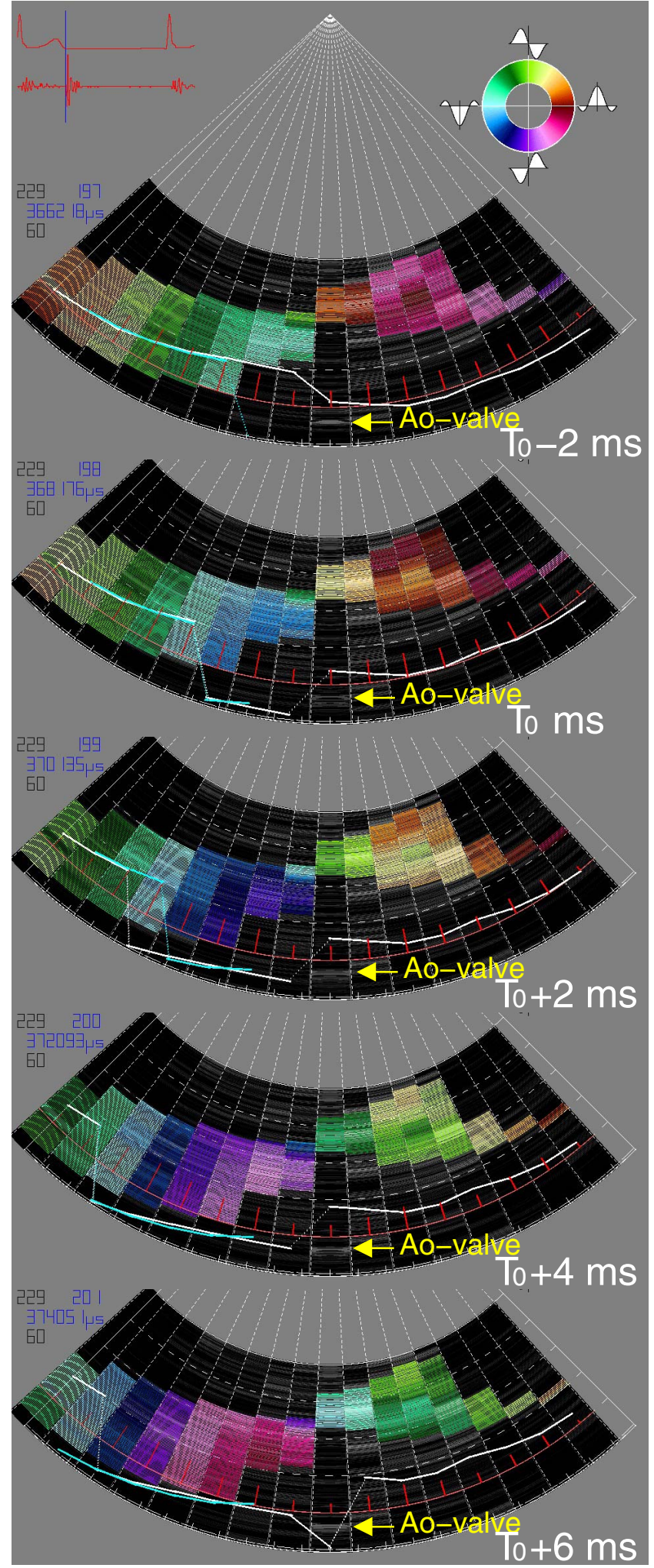

Fig. 2. Spatial distribution of color-coded phase values for $60 \mathrm{~Hz}$ component of the measured wavelets at end-systole of the subject in Fig. 1 consecutively from $T_{0}-2 \mathrm{~ms}$ to $T_{0}+6 \mathrm{~ms}$ around the aortic-valve closure timing $T_{0}$. (ECG: electrocardiogram, PCG: phonocardiogram, LV: left ventricle, RV: right ventricle, IVS: interventricular septum, Ao: aorta.) 
the wave at each point in the IVS after the wave was multiplied by the Hanning window with a short length of $35 \mathrm{~ms}$. For example, at a time $t=T_{0}-2 \mathrm{~ms}$ in Fig. 2, the phase values varied from cyan (+180 degrees) near the root of the $\mathrm{AV}$, through green (+90 degrees), to red (0 degree) at the apexside. This fact shows that for the frequency component $\left(f_{0}\right.$ $=60 \mathrm{~Hz}$ ) of the downward steep impulse around the timing $T_{0}$ in Fig. 1B, the time delay gradually increased from $0 \mathrm{~ms}$ near the root of the $\mathrm{AV}$ to $+9 \mathrm{~ms}\left(=+180 /\left(360 \times f_{0}\right)\right)$ near the apex-side. Moreover, from these consecutively obtained crosssectional 2-D images in Fig. 2, a motion picture can be shown for each frequency component. As shown in the consecutively obtained figures in Fig. 2 and the motion picture, just around the time of AV closure, a few impulses are radiated from the root of AV and propagated along the IVS. The delay due to the propagation of the steep impulse from the root to the apexside is several milliseconds, which has not been recognized at all by any other clinical technique.

The instantaneous phase of the impulse at the LV-surface of the IVS spatially varied as an almost straight line from the root to the apex-side as shown by a white line in the lefthand side in each panel of Fig. 2. The propagation distance can be measured in the cross-sectional image as in Fig. 1A. By fitting the straight line (the cyan line in Fig. 2) with a constant gradient $k$ [radian $/ \mathrm{m}]$ to the spatial distribution of the measured phase, the instantaneous phase velocity $v_{p}[\mathrm{~m} / \mathrm{s}]$ for the frequency component $f_{0}$ was determined by

$$
v_{p}=\frac{2 \pi f_{0}}{k} .
$$

Just at the AV closure timing $T_{0}$, for example, the instantaneous phase velocities $\left\{v_{p}\right\}$ of the 27,60 , and $87 \mathrm{~Hz}$ components of the impulse were, respectively, 2.0, 4.3, and $4.9 \mathrm{~m} / \mathrm{s}$, the order of magnitude of which is the same as those measured for human tissues in the literature [11]-[13], [27]. For each timing of each frequency component, by analysing the phase of the wavelets, the instantaneous phase velocity was measured as in Fig. 3. From end-systole to the beginning of the IR period, there was dispersion among the instantaneous phase velocities, and they rapidly decreased for all frequency components of $10-100 \mathrm{~Hz}$.

\section{Deternimation of Instantaneous Viscoelasticity}

In the parasternal longitudinal-axis view of Fig. 2, the direction of each ultrasonic beam is almost perpendicular to the IVS, and the vibration is detected in the direction along each ultrasonic beam. As shown in Fig. 2, the propagation direction is almost perpendicular to the direction of the detected vibration. This phenomenon is explained by a Lamb wave-a shear vertical wave of the guided wave [21], [22] in a thin wall (IVS) with a thickness of $2 d$. The theoretical phase velocity $v_{p}$ of the Lamb wave at the frequency $f_{0}$ is given by [22]

$$
v_{p}=\sqrt[4]{\frac{4 \mu(\lambda+\mu)}{3 \rho(\lambda+2 \mu)}} \sqrt{2 \pi f_{0} d},
$$

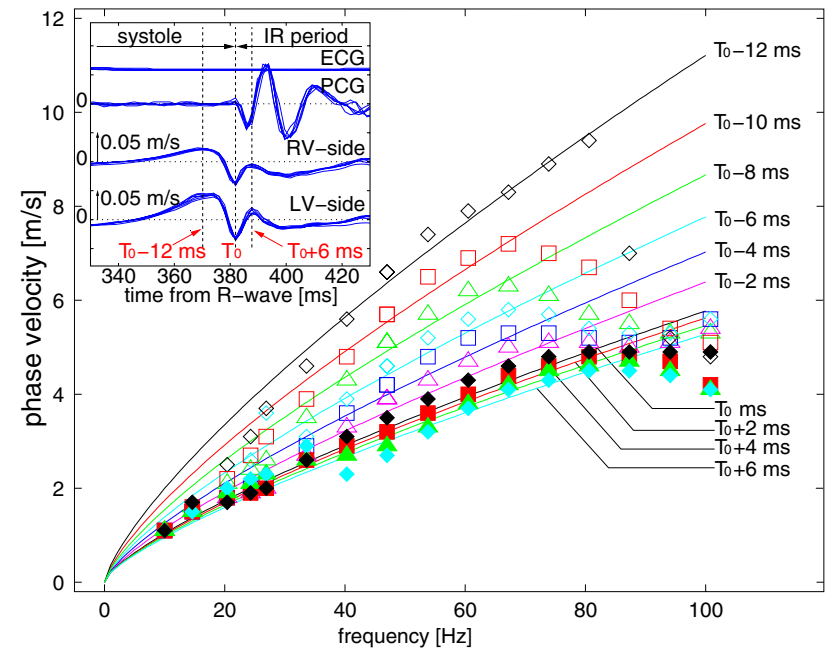

Fig. 3. Measured instantaneous phase velocity values $\left\{v_{p}\right\}$ and fitted theoretical lines of Eq. (3) for frequency components from $10 \mathrm{~Hz}$ to $100 \mathrm{~Hz}$ during the period from $T_{0}-12 \mathrm{~ms}$ to $T_{0}+6 \mathrm{~ms}$ in Fig. 1B. The upper left figure again shows the four waveforms in Fig. 1B but after expansion in the time axis from end-systole to the beginning of the isovolumic relaxation (IR) period. (ECG: electrocardiogram, PCG: phonocardiogram, RV: right ventricle, LV: left ventricle)

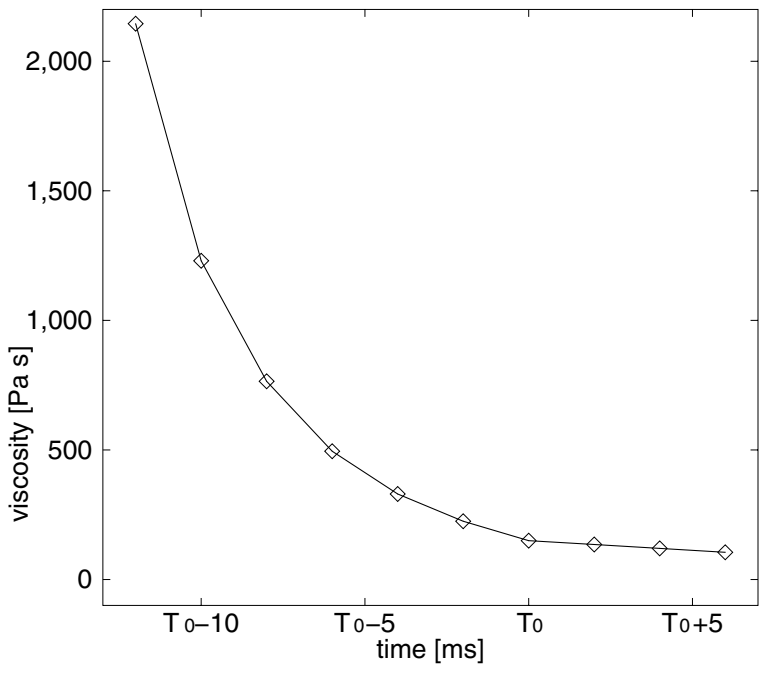

Fig. 4. Transient of the viscosity parameters $\left\{\mu_{2}\right\}$ measured during the period from $T_{0}-12 \mathrm{~ms}$ to $T_{0}+6 \mathrm{~ms}$ in Fig. 3 .

where $\rho$ is the density of the tissue, $\mu$ and $\lambda$ are the Lamè's constants showing the coefficients of shear viscoelasticity and volume viscoelasticity, respectively. From the experimental results [27], $\lambda \gg \mu$. By assuming a simple Voigt model with one spring and one dash-pot in the measured frequency range up to $100 \mathrm{~Hz}$, the shear viscoelasticity parameter $\mu$ is given by a complex value, that is, $\mu=\mu_{1}+j 2 \pi f_{0} \mu_{2}$. Thus, the theoretical value of the phase velocity $v_{p}$ of Eq. (2) is approximated by

$$
v_{p}=\sqrt[4]{\frac{4}{3 \rho}} \Re\left[\sqrt[4]{\mu_{1}+j 2 \pi f_{0} \mu_{2}}\right] \sqrt{2 \pi f_{0} d}
$$


where $\Re[\cdot]$ denotes the real part, $2 d$ is about $10 \mathrm{~mm}$ and can be measured in the cross-sectional image of Fig. 1, and $\rho$ is assumed to be $1.1 \times 10^{3} \mathrm{~kg} / \mathrm{m}^{3}$. By fitting Eq. (3) to the measured instantaneous phase velocities $\left\{v_{p}\right\}$ from 10 to 100 $\mathrm{Hz}$ in Fig. 3, the instantaneous elasticity parameter $\mu_{1}$ and viscosity parameter $\mu_{2}$ were determined. As shown in Fig. $\mathbf{3}$, the phase velocity $v_{p}$ approached zero for low frequency $f_{0}$. Thus, the shear elasticity $\mu_{1}$ in Eq. (3) was negligibly small. The shear viscosity $\mu_{2}$ corresponds to the gradient of the dispersion curve. Figure 4 shows the transient of the viscosity parameters $\left\{\mu_{2}\right\}$ determined for each timing from $T_{0}-12 \mathrm{~ms}$ to $T_{0}+6 \mathrm{~ms}$ in Fig. 3. For end-systole to the beginning of the IR period, the instantaneous viscosity of the myocardium rapidly decreased from about $2.1 \mathrm{kPa} \cdot \mathrm{s}$ to $0.1 \mathrm{kPa} \cdot \mathrm{s}$. This would be due to the rapid decrease in the LV inner pressure from about 120 to several $\mathrm{mmHg}$, which is caused by relaxation of the myocardium. These viscosity values have same order of magnitude as those measured for canine hearts [28].

\section{CONCLUSIONS}

To conclude, we measured rapid and minute vibration signals, which differ from electrically excited waves, simultaneously at multiple points in the IVS to image the wavefront propagation along the heart wall. For the IR period, clear propagation of the steep impulses from the root of the AV to the apex-side was recognized for the first time. From the dispersion of their phase velocities, the viscosity parameter was determined noninvasively. This method offers potential for in vivo imaging of the spatial distribution of the regional viscoelasticity in the myocardium and its rapid change during the IR period, which enable direct assessment of diastolic function based on myocardial relaxation for heart failure [29], [30].

\section{REFERENCES}

[1] E. A. Zerhouni, D. M. Parish, W. J. Rogers, A. Yang, and E. P. Shapiro, "Human heart: tagging with MR imaging - a method for noninvasive assessment of myocardial motion," Radiology, vol. 169, pp. 59-63, 1988.

[2] L. Axel, R. C. Goncalves, and D. Bloomgarden, "Regional heart wall motion: two-dimensional analysis and functional imaging with MR imaging," Radiology, vol. 183, no. 3, pp. 745-750, 1992.

[3] L. T. Mahoney, W. Smith, M. P. Noel, M. Florentine, D. J. Skorton, and S. M. Collins, "Measurement of right ventricular volume using cine computed tomography," Invest. Radiol., vol. 22, no. 6, pp. 451-455, 1987.

[4] A. D. Fleming, X. Xia, W. N. McDicken, G. R. Sutherland, and L. Fenn, "Myocardial velocity gradients detected by Doppler imaging," $\mathrm{Br}$. J. Radiol., vol. 67, no. 799, pp. 679-688, 1994.

[5] R. S. Adler, J. M. Rubin, P. H. Bland, and P. L. Carson, "Quantitative tissue motion analysis of digitized M-mode images: gestational differences in fetal lung," Ultrasound Med. Biol., vol. 16, no. 6, pp. 561-569, 1990.

[6] G. R. Sutherland, C. M. Moran, A. D. Fleming, F. J. Guell-Peris, R. A. Riemersma, L. N. Fenn, K. A. A. Fox, and W. N. McDicken, "Color Doppler myocardial imaging: a new technique for the assessment of myocardial function.," J. Am. Soc. Echocardiogr., vol. 7, pp. 441-458, 1994.

[7] A. Heimdal, A. Støylen, H. T. Drtechn, and T. Skjærpe, "Real-time strain rate imaging of the left ventricle by ultrasound," J. Am. Soc. Echocardiogr., vol. 11, pp. 1013-1019, 1998.

[8] M. Uematsu, K. Miyatake, N. Tanaka, H. Matsuda, A. Sano, N. Yamazaki, and M. Hirama, "Myocardial velocity gradient as a new indicator of regional left ventricular contraction: detection by a two-dimensional tissue Doppler imaging technique," J. Am. Coll. Cardiol., vol. 26, no. 1, pp. 217-223, 1995.
[9] P. Palka, A. Lange, A. D. Fleming, L. N. Fenn, K. P. Bouki, T. R. D. Shaw, K. A. A. Fox, and W. N. McDicken, "Age-related transmural peak mean velocities and peak velocity gradients by Doppler myocardial imaging in normal subjects," Eur. Heart J., vol. 17, pp. 940-950, 1996.

[10] J. Goecsan III, V. K. Gulati, W. A. Mandarino, and W. E. Katz, "Colorcoded measures of myocardial velocity throughout the cardiac cycle by tissue Doppler imaging to quantify regional left ventricular function," $\mathrm{Am}$. Heart J., vol. 131, pp. 1203-1213, 1996.

[11] Y. Yamakoshi, J. Sato, and T. Sato, "Ultrasonic imaging of internal vibration of soft tissue under forced vibration," IEEE Trans. Ultrason., Ferroelect., Freq. Contr., vol. 37, no. 2, pp. 45-53, 1990.

[12] S. F. Levinson, M. Shinagawa, and T. Sato, "Sonoelastic determination of human skeletal muscle elasticity," J. Biomechanics, vol. 28, pp. 1145$1153,1995$.

[13] S. Catheline, J-L. Thomas, F. Wu, and M. A. Fink, "Diffraction field of a low frequency vibration in soft tissues using transient elastography," IEEE Trans. Ultrason., Ferroelect., Freq. Contr., vol. 105, pp. 2941-2950, 1999.

[14] S. Catheline, F. Wu, and M. Fink, "A solution to diffraction biases in sonoelasticity: The acoustic impulse technique," J. Acoust. Soc. Am., vol. 46, pp. 1013-1019, 1999.

[15] L. Sandrin, M. Tanter, S. Catheline, and M. Fink, "Shear modulus imaging with 2-D transient elastography," IEEE Trans. Ultrason., Ferroelect., Freq. Contr., vol. 49, no. 4, pp. 426-435, 2002.

[16] V. Dutt, R. R. Kinnick, and J. F. Greenleaf, "Acoustic shear wave displacement using ultrasound," 1996 IEEE Ultrason. Sympo. Proc., pp. 1185-1188, 1996

[17] S. Chen, M. Fatemi, and J. F. Greenleaf, "Complex stiffness quantification using ultrasound stimulated vibrometry," 2003 IEEE Ultrason. Sympo. Proc., pp. 941-944, 2003.

[18] O. Anosov, S. Berdyshev, I. Khassanov, M. Schaldach, and B. Hensel, "Wave propagation in the atrial myocardium: Dispersion properties in the normal state and before fibrillation," IEEE Trans. Biomed. Eng., vol. 49, no. 12, pp. 1642-1645, 2002.

[19] S. P. Thomas, J. P. Kucera, L. Bircher-Lehmann, Y. Rudy, J. E. Saffits, A. G. Kléber, "Impulse propagation in synthetic strands of neonatal cardiac myocytes with generally reduced levels of connexin43," Circ. Res., vol. 92, pp. 1209-1216, 2003.

[20] H. Kanai, and Y. Koiwa, "Myocardial rapid velocity distribution," Ultrasound Med. Biol., vol. 27, no. 4, pp. 481-498, 2001.

[21] H. F. Tiersten, Linear Piezoelectric Plate Vibrations New York: Prenum Press, 1969.

[22] I. A. Viktorov, Rayleigh and Lamb Waves New York: Prenum Press, 1967.

[23] H. Kanai, M. Sato, Y. Koiwa, and N. Chubachi, "Transcutaneous measurement and spectrum analysis of heart wall vibrations," IEEE Trans. Ultrason., Ferroelect., Freq. Contr., vol. 43, no. 5, pp. 791-810, 1996.

[24] H. Kanai, H. Hasegawa, N. Chubachi, Y. Koiwa, and M. Tanaka, "Noninvasive evaluation of local myocardial thickening and its colorcoded imaging," IEEE Trans. Ultrason., Ferroelect., Freq. Contr., vol. 44, no. 4, pp. 752-768, 1997.

[25] H. Kanai, Y. Koiwa, and J. Zhang, "Real-time measurements of local myocardium motion and arterial wall thickening," IEEE Trans. Ultrason., Ferroelect., Freq. Contr., vol. 46, no. 5, pp. 1229-1241, 1999.

[26] H. Kanai, S. Yonechi, I. Susukida, Y. Koiwa, H. Kamada, and M. Tanaka, "Onset of pulsatile waves in the heart walls at end-systole," Ultrasonics vol. 38, no. 1, pp. 405-411, 2000.

[27] H. L. Oestreicher, "Field and impedance of an oscillating sphere in a viscoelastic medium with an application to biophysics," J. Acoust. Soc. Am., vol. 23, no. 6, pp. 707-714, 1951.

[28] G. H. Templeton, and L. R. Nardizzi, "Elastic and viscous stiffness of the canine left ventricle," J. Appl. Physiol., vol. 36, no. 1, pp. 123-127, 1974.

[29] T. J. Fraites Jr., A. Saeki, and D. A. Kass, "Effect of altering filling pattern on diastolic pressure-volume curve," Circulation, vol. 96, pp. 4408-4414, 1997

[30] S. R. Solomon, and S. A. Glantz, "Regional ischemia increases sensitivity of left ventricular relaxation to volume in pigs," Am. J. Physiol., vol. 276, pp. H1994-H2005, 1999. 